\title{
Patient satisfaction with inpatient care provided by the Sydney Gynecological Oncology Group
}

\author{
This article was published in the following Dove Press journal: \\ Patient Related Outcome Measures \\ 23 November 2010 \\ Number of times this article has been viewed
}

\section{Vivek Arora \\ Shannon Philp \\ Kathryn Nattress \\ Selvan Pather \\ Christopher Dalrymple \\ Kenneth Atkinson \\ Sofia Smirnova \\ Stephen Cotterell \\ Jonathan Carter \\ Sydney Gynecological Oncology Group, Royal Prince Alfred Hospital, University of Sydney, Sydney, Australia}

Correspondence: Vivek Arora

Sydney Gynecological Oncology Group, Level 6, Sydney Cancer Centre, Royal

Prince Alfred Hospital, Missenden Road, Camperdown, New South Wales 2050,

Australia

Tel +6 I 2 95 I56 I I I

Fax +6I 295158434

Email vbbarora@yahoo.com
Purpose: Patient satisfaction with the provision of hospital oncology services can have a significant impact on their overall treatment experience.

Aims: To assess patient satisfaction with the inpatient hospital services in the gynecological oncology setting using the IN-PATSAT32 questionnaire developed by the European Organization for Research and Treatment of Cancer (EORTC).

Methods: A modified version of the IN-PATSAT32 questionnaire with additional 16 items was administered to 52 adult surgical inpatients admitted with the Sydney Gynecological Oncology Group. All participants were provided with an information leaflet regarding the survey and written consent obtained.

Results: A high response rate (100\%) from patients with varied social, ethnic, and educational backgrounds confirmed the acceptability of the survey. Standard of medical care provided, frequency of doctors' visits, exchange of information with doctors, friendliness of the staff, and state of the room ranked highly $(>95 \%)$ on the patient satisfaction scales. Problems were identified with ease of access to and within the hospital, quality of food, and exchange of information with other hospital staff.

Conclusions: Overall the satisfaction with inpatient care was rated very highly in most areas. Deficiencies in certain elements of provision of medical care to the patients were identified and steps have been taken to improve upon these shortcomings.

Keywords: patient satisfaction, EORTC, IN-PATSAT32, gynecological oncology, survey

\section{Introduction}

The Sydney Gynecological Oncology Group at the Sydney Cancer Centre, Royal Prince Alfred Hospital provides outpatient gynecological oncology, colposcopy and pre-admission services as well as inpatient gynecological oncology services. A detailed evaluation of patient satisfaction with the current services provided was initiated in order to identify areas where inpatient services could be improved.

\section{Methods}

Approval was granted by the hospital Ethics Committee to carry out the survey. Between September 2006 and March 2008 surgical inpatients on ward 7E2 were invited to participate in the survey of patient satisfaction. Patients requiring more than an overnight stay in the hospital were randomly selected for the survey. A senior nurse in the gynecological oncology team explained the survey to the patients who were willing to participate. A Participant Information Sheet was provided and written consent obtained from the patients participating in the survey. Patient details for each 
survey were anonymized. The completed surveys were placed in a sealed container and collected by the group's data manager for compilation.

\section{Survey}

The European Organization for Research and Treatment of Cancer (EORTC) IN-PATSAT32 questionnaire was used for the purpose of the survey after completing a User's Agreement. This is a 32-item satisfaction with care questionnaire to measure patients' appraisal of hospital doctors and nurses as well as aspects of care organization and services. The tool has been validated internationally and has been widely used for evaluation of oncology services and to highlight aspects of care needing improvement in an oncology setting. It addresses technical competence, information provision, interpersonal skills, availability, waiting time, access, comfort, and overall care perception. The IN-PATSAT32 is a stand-alone questionnaire addressing the contribution of patient satisfaction with oncology-relevant aspects of care, in the comparison of treatment, psychosocial intervention, or the health care services across countries from various sociocultural backgrounds.

The questionnaire uses a 'poor', 'fair', 'good', 'very good', or 'excellent' response scale to rate each aspect of care. The content of the 32-item questionnaire comprises 11 multi-item scales and three single-item scales, appraising: doctors' technical skills (items 1-3), interpersonal skills (items 4-6), information provision (items 7-9), availability (items 10-11); nurses' interpersonal skills (items 15-17), technical skills (items 12-14), information provision (items 18-20), availability (items 21, 22); other hospital personnel's kindness, helpfulness, information provision (items 24-26); waiting times for medical tests, treatment, and receiving test results (items 27, 28); access to hospital (items 29, 30); exchange of information (item 23); comfort and cleanliness (item 31); and general satisfaction (item 32).

Sixteen additional questions developed by the group were included in the survey. These included questions on efficiency of admissions and nursing staff; timely administration of medication and pain control; information on diet and quality of food; consideration and support to the family; and follow-up arrangements. These questions were developed and included in the survey based on the feedback received from our patients. It was hypothesized that these aspects of inpatient care not included in the IN-PATSAT32 questionnaire could possibly affect the overall inpatient experience in the hospital.
The analysis was carried out with and without the results of the 16 additional questions. The first analysis included a summation of the additional 16 questions to the IN-PATSAT32 survey. The results were then analyzed according to the IN-PATSAT32 methodology; for the multi-item scales, the raw scores for the individual items are summed and then divided by the number of items on the scale. These scales are then linearly transformed with a range from 0 to 100 , a higher scale score representing a higher level of satisfaction.

\section{Results}

During the study period 52 inpatients completed the survey. Thirty-five women (67\%) were married or in a de facto relationship, 6 (12\%) widowed, 5 (10\%) divorced, 5 (10\%) never married, and 1 classified as 'other'. Fifteen (29\%) had attained an education up to year 10, 12 (23\%) year 12/HSC, $10(19 \%)$ a TAFE/Diploma, $10(19 \%)$ a University degree, and $5(10 \%)$ a higher degree. Thirteen patients $(25 \%)$ had some form of medical training, mostly nursing, 30 (58\%) were born in Australia, and 17 (33\%) spoke another language other than English. Thirty-seven patients $(71 \%)$ had children and $3(6 \%)$ patients admitted to a major psychiatric illness.

For the purpose of analysis, the responses rated as 'excellent', 'very good', and 'good' were summed up as 'satisfied' or 'satisfactory' with service provided. The responses 'fair' and 'poor' were deemed as 'unsatisfactory' or 'dissatisfied'. This was based on the assumption that responses rated as 'good' or above indicate a superior quality of service provision and hence were grouped together for the simplicity of analysis.

Summing up the responses rated as 'satisfactory', for the survey questions and additional 16 questions, the following shows in descending order the top responses: 1) the frequency of doctors' visits and consultations; 2) the standard of medical care provided; 3 ) the friendliness and efficiency of the admission staff; and 4) the state and availability of the room (Figure 1).

Those responses rated as 'unsatisfactory' were summed and ranked again for the survey questions and the additional 16 questions are as follows: 1) the ease of access (parking, means of transport); 2) the quality of the food provided; 3 ) the ease of finding one's way to the different departments; and 4) the promptness in answering buzzer calls (Figure 2).

Overall satisfaction with the care received, doctors' availability and their interpersonal skills, exchange of information 


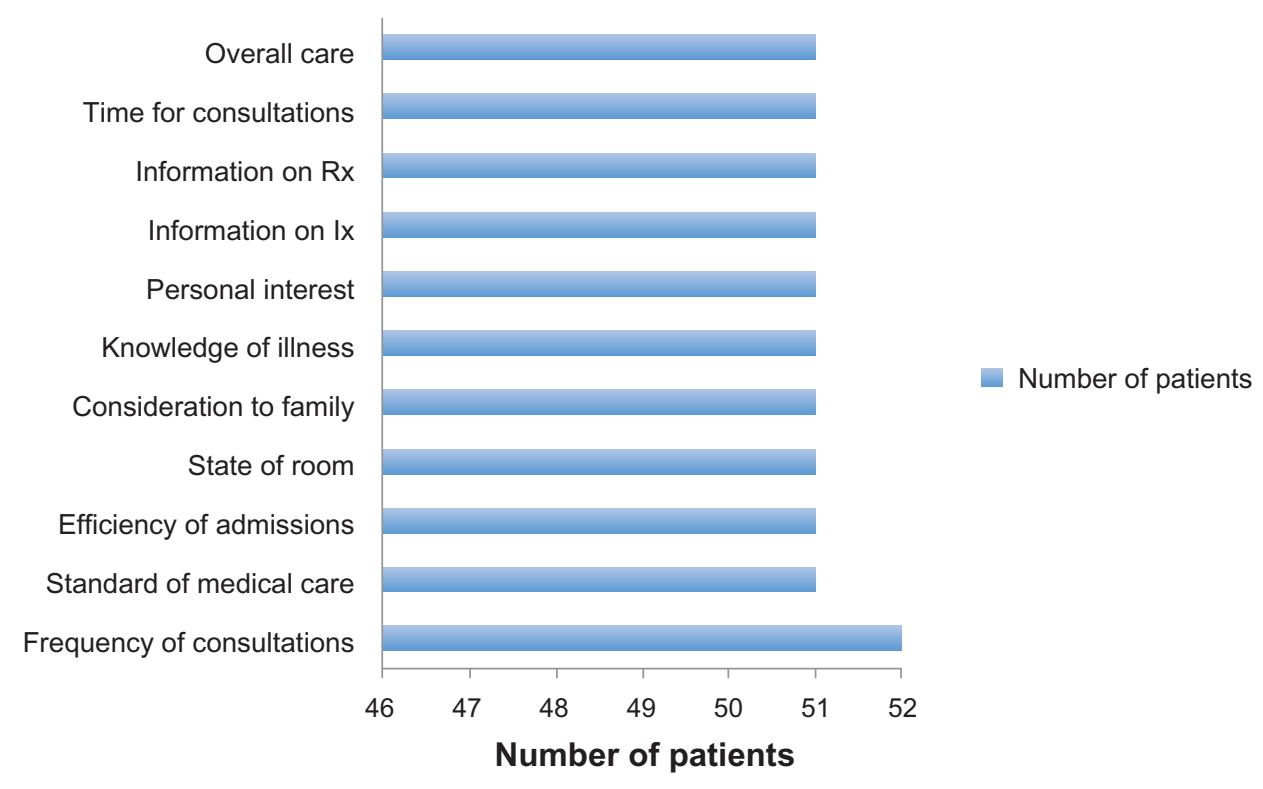

Figure I Top services ranked as satisfactory (>95\%).

Abbreviations: $\mathrm{Rx}$, treatment; Ix, investigations.

with doctors, and their technical skills ranked highest in the IN-PATSAT32 scaled scores (Figure 3).

Access to hospital, helpfulness of other hospital staff, and exchange of information with them, as well as waiting times for investigations and treatment, rated the lowest IN-PATSAT32 scores (Figure 3).

\section{Discussion}

Patient satisfaction has been identified as an important benchmark in health care provision against which hospitals and health care providers are likely to be compared in the future. EORTC INPATSAT32 is a validated patient satisfaction questionnaire that has been used in various oncology settings in differing cultural and socioeconomic settings. We intend to highlight the importance of administering these questionnaires and carrying out patient satisfaction surveys to identify patient needs and to highlight areas of health care provision where improvements can be made. Some deficiencies in health care provision are not immediately apparent either to the providers or to the administrators, and questionnaires like these are an important way of drawing attention to those aspects of health care provision.

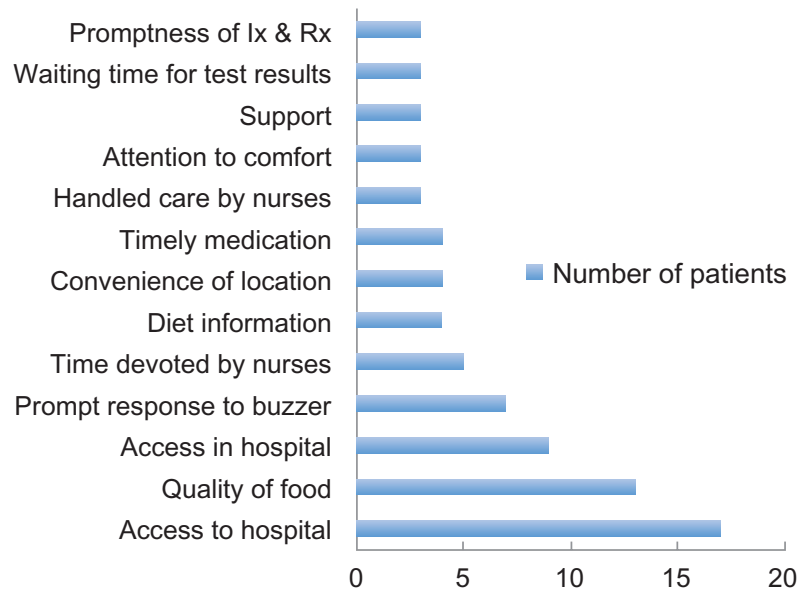

Figure 2 Services ranked as unsatisfactory ( $>5 \%$ )

Abbreviations: $\mathrm{Rx}$, treatment; lx, investigations. 


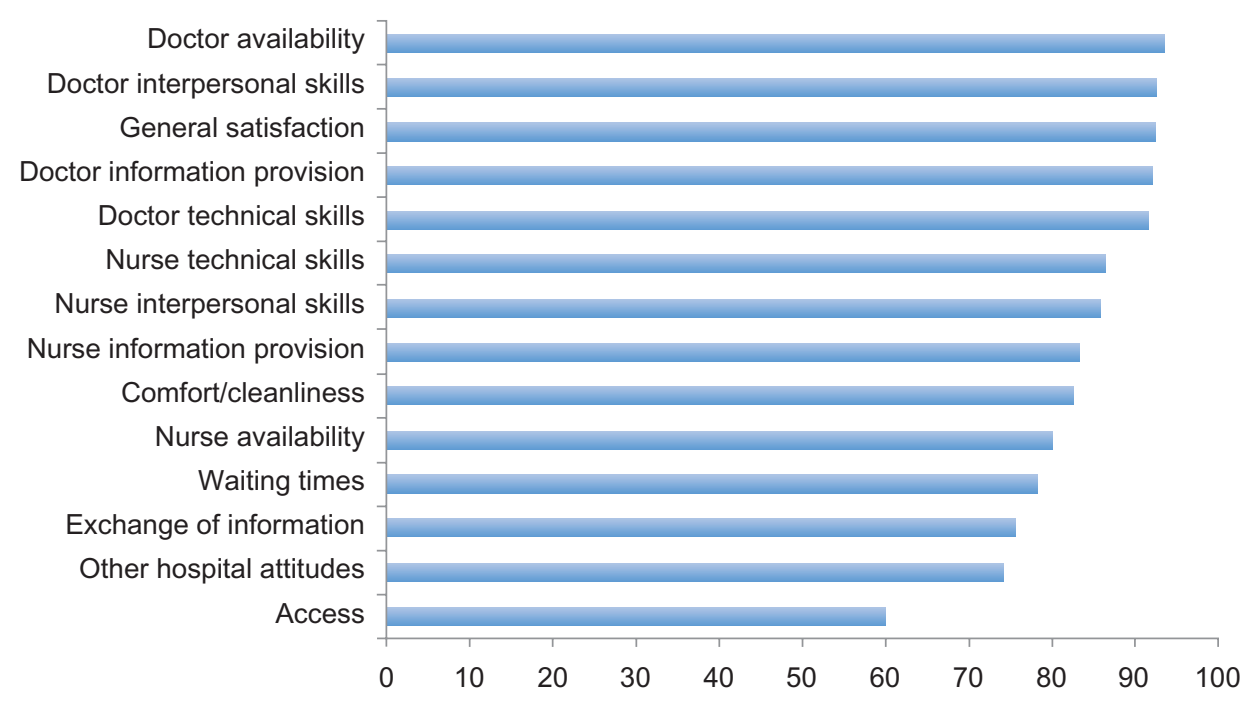

Figure 3 IN-PATSAT32 scaled scores according to category.

The 32-item IN-PATSAT questionnaire developed by the EORTC has been validated and used internationally with success to assess the patient satisfaction with the inpatient care provided at various oncology units. ${ }^{1}$ The survey has a high level of acceptability amongst patients because of ease of administering. It has also shown to be able to discriminate clearly between patients with differing care expectations. The IN-PATSAT32 questionnaire has been validated in various other languages and has demonstrated applicability in varying sociocultural backgrounds. ${ }^{2}$ The IN-PATSAT32 questionnaire covers a comprehensive range of service provision in hospitals. Information provision at various levels figures prominently in this quality assessment questionnaire.

In a follow-up study on the use of the IN-PATSAT questionnaire in the setting of thoracic oncology, the administration of this survey resulted in an overall improvement inpatient satisfaction with the care provided. ${ }^{3}$ In another study where the quality of life questionnaire (QOL-C30) was administered along with the QLQ-PATSAT32 questionnaire to assess patient satisfaction, it was found that the satisfaction rates with hospital care received were independent of the final treatment outcome. ${ }^{4}$

Patient satisfaction is the most widely used outcome measure to assess the doctors' communication skills. ${ }^{5}$ In our survey there was a high level of satisfaction with all aspects of care provided by the doctors, including the exchange of information, frequency of visits, and their technical skills. We added additional 16 questions on aspects of care not covered by the EORTC INPATSAT32 questionnaire based on the feedback received from our patients in the past. It was hypothesized that feedback on these aspects of care would provide information to be forwarded to the hospital administration as well as providing a baseline standard against which to compare results from future surveys on patient satisfaction.

Although deficiencies were identified in certain aspects of nursing care provided at the hospital, the overall scores are better than those reported in another study carried out in a similar setting. ${ }^{3}$ Steps have been taken since the administration of the survey to address these issues. Other areas where improvements can be made are exchange of information with other hospital staff, interpersonal skills and attitudes of other hospital staff, waiting times, and access to the hospital. The findings of our study are similar to those identified by other authors, ${ }^{3}$ and this information has been passed on to relevant authorities. We hope that availability of objective information will lead to increased hospital investment in these areas.

The scores obtained from the EORTC INPATSAT32 questionnaire serve as a benchmark against which to compare the results from any future surveys. We are currently in the process of carrying out a follow-up survey to assess the impact of administering the satisfaction survey to our gynecological oncology inpatients. The questionnaire has been used in various surgical and medical oncology settings to evaluate patient satisfaction. To the best of our knowledge, after a thorough review of English medical literature, the questionnaire has not been used in the setting of gynecological oncology inpatient care. 


\section{Conclusion}

Overall the satisfaction with inpatient care was rated very highly in most areas. Persistent issues of poor satisfaction relate to food, access, and parking, which by and large are out of the control of the attending nurses, doctors, and other hospital staff. Further effort needs to be directed into these areas to improve overall patient satisfaction. A follow-up survey is being carried out to evaluate the impact of the changes introduced on the provision of gynecological oncology services at our center.

\section{Disclosure}

The authors report no conflicts of interest in this work.

\section{References}

1. Bredart A, Bottomley A, Blazeby JM, et al. An international prospective study of the EORTC cancer in-patient satisfaction with care measure (EORTC IN-PATSAT32). Eur J Cancer. 2005;41(Pt 14):2120-2131.

2. Jayasekara H, Rajapaksa L, Bredart A. Psychometric evaluation of the European Organisation for Research and Treatment of Cancer in-patient satisfaction with care questionnaire ('Sinhala' version) for use in South Asian setting. Int J Qual Health Care. 2008;20(Pt 3):221-226.

3. Leo F, Radice D, Didier F, et al. Does a personalised approach improve patient satisfaction in thoracic oncology? Am J Manag Care. 2009; 15(Pt 6):361-367

4. Aver KN, Metcalfe C, Nicklin J, et al. Satisfaction with care: an independent outcome measure in surgical oncology. Ann Surg Oncol. 2006; 13(Pt 6):764-765.

5. Ong LM, de Haes JC, Hoos AM, Lammes FB. Doctor-patient communication: a review of literature. Soc Sci Med. 1995;40:903-918. 


\section{Appendix I Items of EORTC IN-PATSAT32 Questionnaire}

Scoring procedure for the EORTC IN-PATSAT32'

The international field-testing study of the EORTC cancer inpatient satisfaction with care measure (EORTC INPATSAT32) has confirmed the hypothetical structure of the questionnaire. This questionnaire should thus be scored as follows:

1. Content of the questionnaire

Multi-item scales

- Doctors

Interpersonal skills (items 4-6)

Technical skills (items 1-3)

Information provision (items 7-9)

Availability (items 10, 11)

- Nurses

Interpersonal skills (items 15-17)

Technical skills (items 12-14)

Information provision (items 18-20)

Availability (items 21, 22)

- Other hospital personnel; kindness, helpfulness, and information giving (24-26)

- Waiting time; performing medical tests/treatment, receiving medical tests results (items 27, 28)

- Access (items 29, 30)

Single-item scales

- Exchange of information (item 23)

- Comfort/cleanness (item 31)

- General satisfaction (item 32)

2. Format of the questionnaire

Period of reference: Refer to interactions with health care providers and services in the oncology hospital during hospital stay

How would you rate? Poor (1)/Fair (2)/Good (3)/Very Good (4)/Excellent (5)

Number of items: 32
3. Scoring procedure

All multi-item or single-item scales are constructed in a similar manner: 1) the raw scores for the individual items within a scale are first summed, and then, for the multiitem scales, divided by the number of items in the scale; and 2) these scale scores are then linearly transformed such that all scales range from 0 to 100 , with a higher scale score representing a higher level of satisfaction with care.

\section{EORTC IN-PATSAT32}

Copyright: EORTC Quality of Life Group

Available for users at http://groups.eortc.be/qol/question naires_eortcinpatsat32.htm

\section{Appendix 2}

\section{Additional questions asked}

In general how do you rate the following:

- The standard of medical care provided at this hospital?

- The friendliness and efficiency of the admission staff?

- The state and availability of your room?

- The information regarding your diet?

- The quality of the food provided?

- The respect shown for your privacy?

- The respect and consideration shown to your family and support people?

- The efficiency of nursing care?

- Friendliness and courtesy of the staff?

- Convenience of location for you?

- Cost to you?

- Your pain control?

- The amount of information provided to family or support people?

- Timely delivery of medicine?

- Were your follow-up arrangements optimal?

- Level of recomendation of this service to friends and family?
Patient Related Outcome Measures

\section{Publish your work in this journal}

Patient Related Outcome Measures is an international, peer-reviewed, open access journal focusing on treatment outcomes specifically relevant to patients. All aspects of patient care are addressed within the journal and practitioners from all disciplines are invited to submit their work as well as healthcare researchers and patient support groups. Areas covered will

\section{Dovepress}

include: Quality of life scores; Patient satisfaction audits; Treatment outcomes that focus on the patient; Research into improving patient outcomes; Hypotheses of interventions to improve outcomes; Short communications that illustrate improved outcomes; Case reports or series that show an improved patient experience; Patient journey descriptions or research. 